\title{
ZMM proteins during meiosis: Crossover artists at work
}

\author{
Audrey Lynn, Rachel Soucek \& G. Valentin Börner* \\ Department of Biological, Geological and Environmental Sciences, Cleveland State University, Cleveland, \\ OH 44115, USA; Tel: +1-216-523-7557; E-mail: g.boerner@csuohio.edu \\ * Correspondence
}

Key words: double Holliday junctions, meiosis, recombination, stable strand invasion, ZMM proteins

\begin{abstract}
Faithful segregation of homologous chromosomes (homologs) during meiosis depends on chiasmata which correspond to crossovers between parental DNA strands. Crossover forming homologous recombination takes place in the context of the synaptonemal complex (SC), a proteinaceous structure that juxtaposes homologs. The coordination between molecular recombination events and assembly of the SC as a structure that provides global connectivity between homologs represents one of the remarkable features of meiosis. ZMM proteins (also known as the synapsis initiation complex = SIC) play crucial roles in both processes providing a link between recombination and SC assembly. The ZMM group includes at least seven functionally collaborating, yet structurally diverse proteins: The transverse filament protein Zip1 establishes stable homolog juxtaposition by polymerizing as an integral component of the SC. Zip2, Zip3, and Zip4 likely mediate protein-protein interactions, while Mer3, Msh4, and Msh5 directly promote steps in DNA recombination. This review focuses on recent insights into ZMM functions in yeast meiosis and draws comparisons to ZMM-related proteins in other model organisms.
\end{abstract}

\section{Homolog segregation requires connections}

Meiosis is the specialized cell division by which sexually reproducing organisms generate haploid gametes from diploid precursor cells. During meiosis, one round of genome replication is followed by two rounds of chromosome segregation, with homologs segregating during meiosis I, and sister chromatids segregating during meiosis II. Prior to the onset of meiosis I, homologs have to attach to opposite spindle poles. Bipolar homolog attachment depends on chiasmata, cytologically detectable associations between homologs, which, in combination with intersister connections, provide a counterforce to pole-directed spindle forces, thereby ensuring correct positioning of each homolog pair.
Chiasmata correspond to crossovers on the DNA. Crossovers are level recombination events where two nonsister chromatid arms have undergone reciprocal exchange. Crossovers/chiasmata play a key role in faithful homolog segregation. In the absence of crossovers, homologs segregate randomly, resulting in the formation of gametes with a surplus or deficit of an entire chromosome. Fertilization involving gametes with such chromosomal imbalances invariably results in miscarriage or severe birth defects, most prominently Down syndrome in humans (Hassold \& Hunt 2001).

Events on the DNA level and assembly of global chromosome structures occur during the prophase of meiosis I. They are temporally and spatially closely coordinated. On the DNA level, programmed double- 


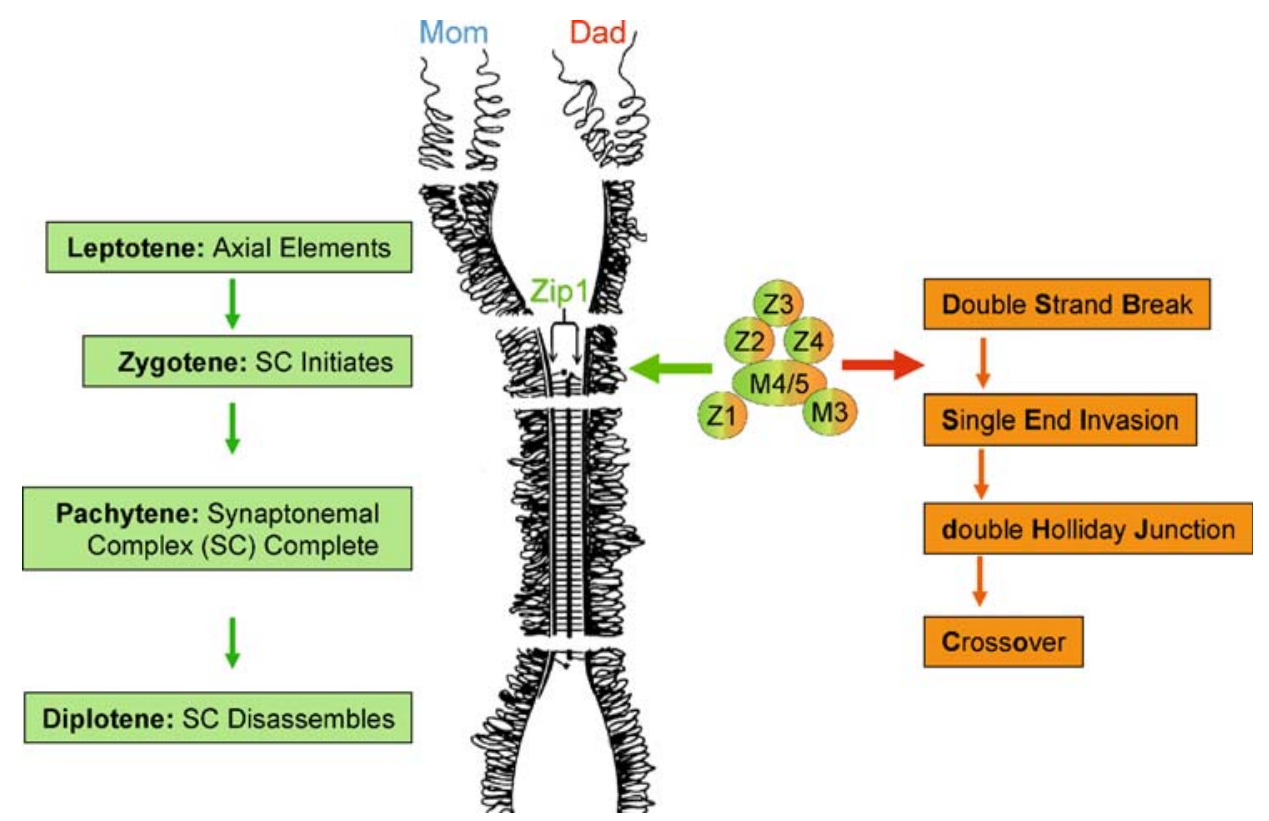

Figure 1. Functions of ZMM proteins in synaptonemal complex assembly and recombination. On the left side, the cytological stages of meiosis I prophase are depicted. On the right side, the intermediate stages of crossover formation are shown. ZMM proteins are Zip3 (Z3), Zip2 (Z2), Zip4/Spo22 (Z4), Msh4/Msh5 (M4/5), Mer3 (M3), Zip1 (Z1). Localization of Zip1 as a transverse filament component of the synaptonemal complex central element is also indicated. (Adapted from Alberts et al. The Molecular Biology of the Cell, 4th edn, Garland Science; 2002).

stranded breaks (DSBs) are formed and a nonrandom subset of them is processed into crossovers with the remainder undergoing noncrossover recombination (see below). On the chromosome structure level, homologs align and become closely juxtaposed along their entire lengths via a proteinaceous structure called the synaptonemal complex (SC; Figure 1).

SC assembly entails three major stages which are also used to classify intermediate stages of meiosis I prophase (Figure 1): During leptotene, axial elements form along the base of sister chromatids. Then, during zygotene, transverse filaments start polymerizing from distinct sites, progressively connecting axial elements of two homologs. During pachytene, when $\mathrm{SC}$ assembly is complete, homologs are closely juxtaposed via a dense array of transverse filaments, constituting the SC central element, flanked by lateral elements, which correspond to the former axial elements (de Boer \& Heyting 2006). SC disassembly occurs at the transition from pachytene to diplotene. It is at this stage that chiasmata become cytologically detectable as connections between homologs.
SC assembly is closely coordinated with homologous recombination on the DNA level. Programmed DSBs are introduced during leptotene at multiple sites. DSBs subsequently undergo rapid resection at their $5^{\prime}$ ends, generating single-stranded $3^{\prime}$ overhangs approximately 600 nucleotides in length (Figure 2; Sun et al. 1989). 3' overhangs then undergo strand exchange with allelic sequences on the homolog. Only a subset of DSBs is processed into crossovers, with the remainder progressing to noncrossovers, i.e., recombination events where chromatid regions flanking the recombination site retain their parental configuration following localized sequence transfer between homologs. Some meiotic DSBs are also repaired via interactions with their sister chromatid (Schwacha \& Kleckner 1997). Importantly, however, noncrossovers or intersister recombination events do not contribute to homolog segregation as they fail to establish physical linkage between homologs.

Differentiation into future crossovers and noncrossovers is now widely believed to occur no later than the time of initial strand exchange (Allers \& 


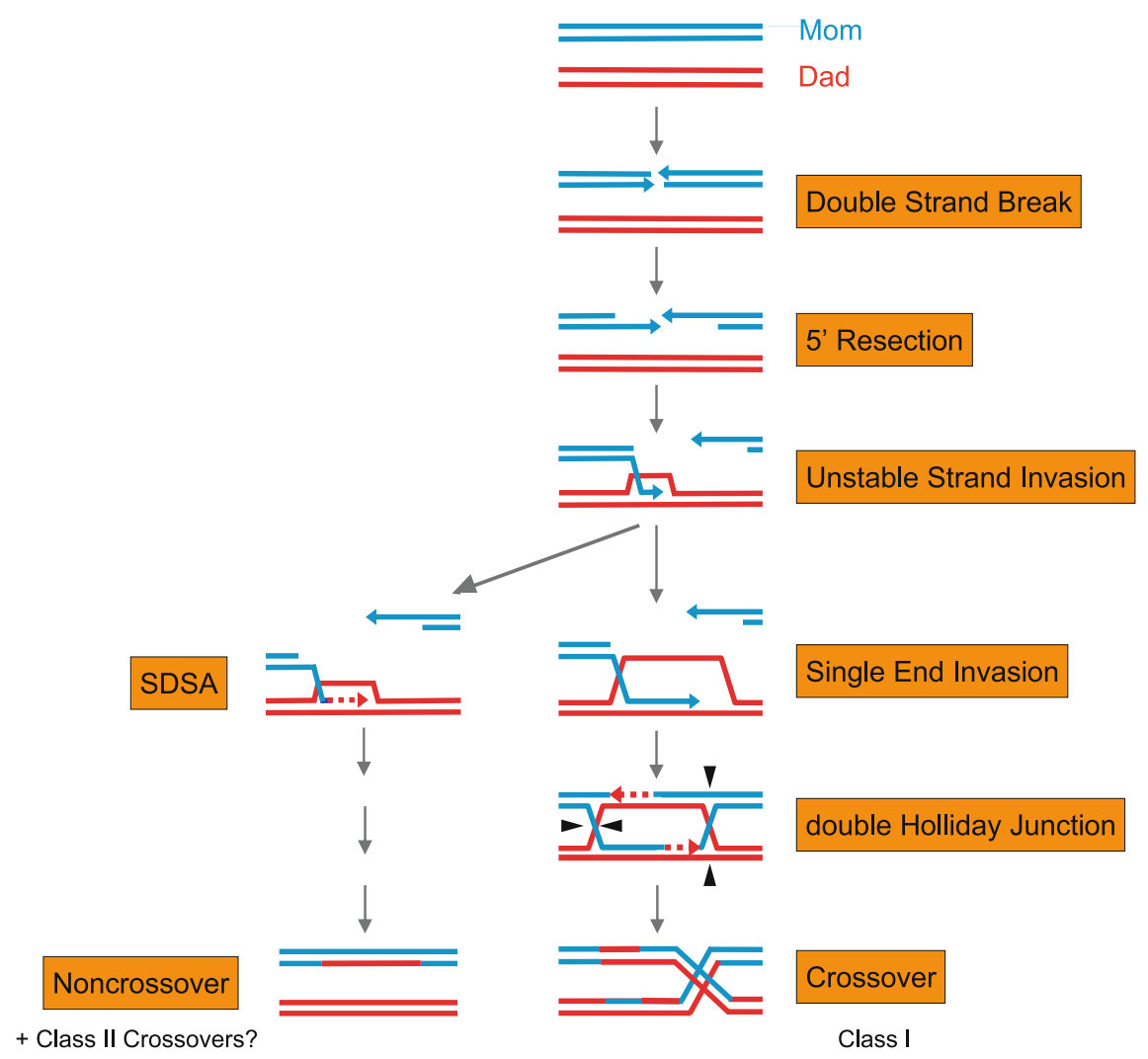

Figure 2. The early decision model of meiotic recombination. Parental double-stranded DNA molecules are depicted in blue and red. Arrows mark $3^{\prime}$ ends. Only the main recombination pathways are shown in detail. SDSA = synthesis-dependent strand annealing. Class I crossovers represent the majority of crossovers formed in yeast and Arabidopsis. They are formed via double Holliday junctions. Class II crossovers form in the absence of double Holliday junctions and may share steps with the noncrossover pathway (Börner et al.2004).

Lichten 2001, Börner et al. 2004). The early decision model suggests that crossover-designated DSBs are processed into crossovers via two intermediates following strand exchange (Figure 2): First, crossover-bound strand invasion events become stabilized, giving rise to single-end invasions (SEI) (Hunter \& Kleckner 2001, Börner et al. 2004, Bishop \& Zickler 2004). This transition occurs at the exit from zygotene. During pachytene, the opposing end of a DSB is captured by the SEI intermediate, followed by religation of the two DSB ends to generate double Holliday junctions (Schwacha \& Kleckner 1995). At or shortly before pachytene exit, double Holliday junctions are resolved into crossovers (Hunter \& Kleckner 2001). At positions where strand invasion fails to become stabilized, noncrossovers are formed by a process referred to as synthesis-dependent strand annealing (SDSA; Allers \& Lichten 2001). In this case, the invading DSB is predicted to undergo $3^{\prime}$ extension in a structure that entails only a short heteroduplex region, followed by ejection from the template strand and religation of the two parental strands.

The genome-wide distribution of crossovers is under twofold control. First, every homolog pair, irrespective of size, receives at least one crossover, but rarely more than two or three crossovers, in most organisms. This low average number of crossovers per meiotic cell necessitates a mechanism to ensure that each homolog pair receives the one crossover obligatory for homolog segregation. Controlled formation of an obligatory crossover is particularly important in organisms with chromosomes that differ extensively in size: In humans, the smallest and largest chromosomes differ 5-fold in length. Despite such differences, both small and large chromosomes acquire at least one crossover and segregate efficiently (Tease et al. 2002). When more than one 
crossover happens along a homolog pair, a crossover in a given chromosome region reduces the probability of additional crossovers nearby. This phenomenon, called positive crossover interference, results in maximum spacing of crossovers along homolog pairs. Interference implies a mechanism of communication between potential crossover sites along a given chromosome (Zickler \& Kleckner 1999). The mechanism(s) controlling crossover interference and obligatory crossover formation are currently not understood. Whether the two levels of control are functionally linked is also unknown.

\section{ZMM protein functions are widely conserved}

The ZMM group (an acronym for yeast proteins Zip1/Zip2/Zip3/Zip4, Msh4/Msh5, Mer3) includes at least seven functionally collaborating yet evolutionarily unrelated proteins. Initially identified in the yeast $S$. cerevisiae as proteins with functions in recombination and SC assembly, presumed ZMM orthologs have also been identified in animals and plants, suggesting evolutionary conservation of this group. Co-localization of ZMM proteins along meiotic yeast chromosomes and similarities among $\mathrm{zmm}$ mutant phenotypes suggest functional collaboration between these proteins.

A common feature of $\mathrm{ZMM}$ proteins in all investigated organisms is their role in crossover formation. Despite efficient initiation of recombination, crossovers are reduced or absent in $\mathrm{zmm}$ mutants. ZMM proteins further play important roles in the assembly of the SC central element/synapsis in organisms where recombination initiation is required for synapsis, e.g., yeast, mouse, and Arabidopsis. zmm mutant phenotypes in different organisms provide insights into specific roles of each protein, and also into the question of how recombination and $\mathrm{SC}$ formation are functionally linked.

Three subgroups of ZMM proteins can be distinguished based on functional criteria: Subgroup I includes highly conserved proteins that exhibit sequence similarities with proteins involved in mitotic DNA metabolism: The predicted yeast Mer3 protein contains seven motifs characteristic of the DExH box type of DNA/RNA helicases (Nakagawa \& Ogawa 1999). Mer3 indeed unwinds various double-stranded substrates in an ATP-dependent manner (Nakagawa et al. 2001). Msh4 and Msh5 are two of the five eukaryotic, nuclear homologs of MutS, a protein that in bacteria scans the DNA for mismatches, binds at the mismatch, and (together with MutL and MutH) forms an active repair complex. Like other MutS homologs, Msh4/Msh5 appear to function as a heterodimer. They perform mostly meiotic functions, and are not required for standard mismatch repair (Hollingsworth et al. 1995). Orthologs of yeast Mer3 have been identified and/or functionally studied in plants (Chen et al. 2005, Mercier et al. 2005) and humans (Tanaka et al. 2006). Orthologs of Msh4/Msh5 have been investigated in C. elegans (Zalevsky et al. 1999, Kelly et al. 2000), mouse (de Vries et al. 1999, Kneitz et al. 2000), and Arabidopsis (Higgins et al. 2004).

Subgroup II includes three proteins, Zip2, Zip3, and Zip4/Spo22, implicated in ubiquitinylation and/ or SUMOylation, likely as a mechanism to modify protein interactions (Perry et al. 2005, Cheng et al. 2006). The three proteins are part of a small evolutionary cohort comprising homologs in related yeasts. An ortholog of Zip3 has so far only been identified in C. elegans (Jantsch et al. 2004). Presumed orthologs of Zip4 have been identified in plants and other higher eukaryotes (Chelysheva et al. 2007)

The founding member of subgroup III is Zip1, a coiled-coil protein with two terminal globular domains. Zip1 localizes along the length of the yeast SC during pachytene and likely corresponds to the transverse filament component of the SC detected by electron microscopy (Sym et al. 1993). Orthologs of Zip1 in the strict sense have not been identified, yet several model organisms contain proteins that localize to the SC and share structural similarities with Zip1, including an extensive central coiled-coil region. These proteins include mammalian SC protein Sycp1 (de Vries et al. 2005), two closely related Arabidopsis proteins called ZYP1a and ZYP1b (Higgins et al. 2005), Syp-1 and Syp-2 in C. elegans (e.g., Colaiacovo et al. 2003), and c3G in Drosophila (see Page \& Hawley 2004). Several excellent reviews have appeared recently covering these transverse filament proteins (Page \& Hawley 2004, de Boer \& Heyting 2006) and we will mostly concentrate on the yeast protein Zip1, which closely interacts with other ZMM proteins. 


\section{ZMM proteins mediate crossover formation}

The formation of functional crossovers depends critically on the ZMM group of proteins in all organisms investigated to date. Details of mutant phenotypes, and the extent to which ZMM proteins are required for crossover formation, vary substantially, however, not only between different organisms but even for the same mutation in a given organism under different conditions.

Meiotic crossover frequencies have traditionally been measured as the frequency at which appropriately spaced (phenotypic or molecular) markers get recombined into nonparental combinations. Such assays are of limited use for analysis of recombination in $\mathrm{zmm}$ mutants, however, since functional gametes are usually not recovered, either due to high levels of chromosome missegregation (in yeast or $C$. elegans) or due to a regulatory arrest during meiosis I prophase (in yeast) and ensuing programmed cell death of the majority of cells (in mouse; e.g., de Vries et al. 1999).

Physical and cytological assays have been developed to measure crossover rates independently of functional gamete formation. Physical analysis monitors recombination on the DNA level by molecular approaches. It depends on the availability of a hotspot of recombination where crossovers occur at high frequencies (typically 1-20\% of parental DNA) in relatively small intervals (typically $<10 \mathrm{~kb}$ ), making DNA molecules accessible to standard agarose gel analysis. Crossovers can be distinguished from parental molecules due to restriction length polymorphisms. An alternative system makes use of a yeast strain in which one homolog is circular, with single and double crossovers generating chromosome dimers or trimers, respectively (Game et al. 1989). Physical analysis has mostly been used in yeast but has also been developed for a mouse system (Guillon et al. 2005).

Cytological assays measure abundance and distribution of chromosomal structures and of proteins associated with ongoing or completed recombination. Chiasmata represent completed crossover events and become detectable at diakinesis, but can be visualized also when premature condensation is experimentally induced in pachytene chromosomes (e.g., Marcon \& Moens 2003). Prior to the identifi- cation of specific proteins associated with crossing over, late recombination nodules were used to monitor emerging crossovers. Late recombination nodules correspond in number and distribution to designated crossover positions (Carpenter 1987). More recently, crossover-specific marker proteins which localize to the SC during pachytene, such as mismatch repair proteins Mlh1 and Mlh3, have been used to determine designated crossover events (Lynn et al. 2004). In wild-type, Mlh1 localizes to chiasmata that have been made visible by inducing premature condensation (Marcon \& Moens 2003). Notably, cytological markers like Mlh1 likely localize to recombination interactions before completion of crossover formation and may thus not represent completed crossover events (see below).

ZMM proteins play an important role in the formation of the majority of crossovers in yeast. Essentially no COs were detected by physical analysis at $30^{\circ} \mathrm{C}$ in a BR strain lacking ZIPl (Sym et al. 1993). Physical analysis in another widely used strain, SK1, at $33^{\circ} \mathrm{C}$ revealed similar defects in crossover formation in zipl $\Delta$, zip2 $\Delta$, zip3 3 , zip $4 \Delta$, $m e r 3 \Delta$, and $m s h 5 \Delta$ : In particular, at one recombination hotspot, $>85 \%$ of crossovers were eliminated (Börner et al. 2004, Perry et al. 2005). Analysis of recombination at a different hotspot of recombination in SK1 at $30^{\circ} \mathrm{C}$ showed elimination of $90-95 \%$ of

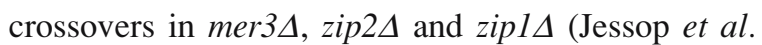
2006). 75 to $80 \%$ of crossovers were eliminated in zip1 $\Delta$, zip3 $\Delta$ and zip $4 \Delta$, respectively, using the circular chromosome construct in the BR strain (Agarwal \& Roeder 2000, Tsubouchi et al. 2006, Mitra \& Roeder 2007). ZMM proteins thus are required for the formation of more than $80 \%$ of crossovers in yeast.

Elimination of the majority of crossovers in yeast $z m m$ mutants is not due to reduced DSB formation: DSBs accumulate to wild-type levels in zmm mutants when assayed in a strain defective for DSB processing (Börner et al. 2004). Normal recombination initiation in $\mathrm{zmm}$ mutants can also be inferred from assays measuring gene conversion following return to mitotic growth conditions (Sym et al. 1993). Moreover, noncrossovers (NCOs) which can also be measured by physical assays form at approximately normal levels and with normal kinetics in various zmm single and double mutants at $30^{\circ} \mathrm{C}$ and $33^{\circ} \mathrm{C}$ 
(Börner et al. 2004, Perry et al. 2005, Jessop et al. 2006). Thus, NCOs form independent of ZMM proteins. Yeast ZMM proteins are specifically required for processing a subset of recombination interactions designated to become crossovers. Differentiation of DSBs into future COs and NCOs occurs also independently of ZMM proteins.

Phenotypes of zmm mutants for CO formation in C. elegans, mouse, and Arabidopsis exhibit numerous similarities with those in yeast, but also a few important differences. In the absence of Msh4, Msh5, or Zip3 homolog Zhp3, almost all homologs are present as univalents rather than bivalents during $C$. elegans diakinesis, consistent with the absence of chiasmata (Zalevsky et al. 1999, Kelly et al. 2000, Jantsch et al. 2004). Crossover rates in viable offspring that form despite high levels of homolognondisjunction, indicate that $>99 \%$ of $\mathrm{COs}$ are eliminated in these mutants. Absence of $C$. elegans transverse filament proteins SYP-1 or SYP-2 similarly eliminates most COs (MacQueen et al. 2002, Colaiacovo et al. 2003).

In mouse mutant strains lacking Msh4 or Msh5, COs are also completely eliminated. Homologs fail to align properly and no chiasmata are detected (de Vries et al. 1999, Edelmann et al. 1999, Kneitz et al. 2000). In male meiosis, cells undergo apoptosis in large numbers before entry into pachytene, thus never reaching the stage when crossover-specific cytological structures normally appear. Defects in $M s h 4^{-1-}, M s h 5^{-1-}$ double mutants are similar to the respective single mutants (Kneitz et al. 2000). In the Sycp1 knockout mouse, neither Mlh1 foci nor chiasmata are detected, again suggesting elimination of CO formation (de Vries et al. 2005). In the absence of Sycp1, markers of early recombination, such as RecA homologs Dmc1/Rad51, Msh4, and eukaryotic single-strand binding protein RPA, occur normally but no Mlh1/3 foci appear at the time of normal pachytene, consistent with normal recombination initiation but a failure to complete $\mathrm{CO}$ formation (de Vries et al. 2005). Msh4, Msh5, and Sycp1 may perform additional functions besides processing of crossover-designated events: Rad51 foci persist at more sites $(>100)$ than expected for COs $(<25)$, consistent with a surplus of recombination initiation, defects in recombination progression at sites not designated as COs, or designation of additional CO sites (Kneitz et al. 2000, de Vries et al. 2005).
Absence of Mer3 (also known as RCK) or Msh4 in Arabidopsis eliminates $>85 \%$ of COs, observable by coordinate reduction of chiasmata and bivalents (Mercier et al. 2005, Higgins et al. 2004, Chen et al. 2005). The Arabidopsis genome encodes duplicated copies of ZYPI ( $a$ and $b$ ) which appear to have largely overlapping functions, such that both genes have to be eliminated to affect $\mathrm{CO}$ formation and synapsis (Higgins et al. 2005). When both ZYPla and $Z Y P 1 b$ are inactivated, only $20 \%$ of COs are eliminated (as measured by chiasma frequencies and Mlh1 foci). Intriguingly, chromosomes are frequently associated with nonhomologous chromosomes in this mutant. In other organisms, transverse filament mutants do not exhibit nonhomologous associations or ectopic COs. Zyp1 may control interhomolog CO formation at the time of partner selection, a process that likely occurs during strand invasion (see below). Given the much less severe effect of a zypla, zyplb double mutant on $\mathrm{CO}$ formation, Zyp $1 \mathrm{~A} / \mathrm{B}$ in Arabidopsis may not collaborate with other ZMM proteins as closely as, for example, observed for Zip1 in yeast. Central element components may affect recombination to various degrees.

Consistent with a direct role in recombination, ZMM proteins are cytologically associated with nascent recombination events. Yeast forms around 90 COs per meiosis per yeast cell (Fung et al. 2004). This corresponds to 5-6 COs along each of the 16 yeast chromosomes, an unusually high number compared to animals and plants in which only one or two COs occur. Yeast ZMM proteins, including Msh4, Zip2, Zip3, and Zip4 localize to the same 60-70 sites along homolog pairs during pachytene (e.g., Rockmill et al. 2003), a number that approximately matches that expected for COs. Strikingly, ZMM foci are nonrandomly distributed just like COs. Thus, a Zip2 focus in a given chromosome region reduces the probability of additional Zip2 foci in adjacent intervals (Fung et al. 2004). ZMM foci may correspond to late recombination nodules in higher organisms, which likely represent crossover-designated sites (Fung et al. 2004, Carpenter 1987).

A close correlation between ZMM foci and COs is supported by studies that examined the effects of reduced DSB levels on Zip3 foci and COs. Strikingly, crossovers and Zip3 foci decline coordinately when DSBs are reduced below a certain threshold, revealing a correlation between COs and Zip3 foci. Above 40\% normal DSBs levels, COs and Zip3 foci 

late time points when wild-type recombination has essentially been completed (Nakagawa \& Ogawa 1999, Börner et al. 2004). At a different hotspot, persisting DSBs are also observed for zip2 $\Delta$ and mer3 $\Delta$ (but not zipl $\Delta$ and $m s h 4 \Delta$ ), possibly due to somewhat different experimental conditions (Jessop et al. 2006). Second, in zip $2 \Delta$, zip $3 \Delta$, $m s h 5 \Delta$, and mer3 $\Delta$, SEIs and dHJs are coordinately reduced or even eliminated (Börner et al. 2004). The same defects also occur in representative double mutants. The zipls mutant deviates somewhat from this pattern, since reduced number of SEIs and dHJs form, but persist and eventually rise to close to wildtype levels (Storlazzi et al. 1996, Börner et al. 2004). Importantly, zipl $\Delta$ exhibits a defect in recombination that is CO-specific and occurs at the transition from DSB to SEIs, just as in other $\mathrm{zmm}$ mutants as indicated by delayed formation of SEIs. Consistent with a role of Zip1 at an early stage of meiotic recombination, Rad51 foci persist at high levels at the point of meiotic arrest in another strain (BR) not accessible to physical analysis (Bishop 1994).

At present physical analysis is not feasible in organisms other than yeast and recombination intermediates cannot be monitored directly. Instead, the recombination status is inferred from abundance and kinetics of cytological markers that likely correlate with certain intermediate recombination stages. Markers include Rad51, gammaH2AX, a histone H2 phosphorylation associated with DSBs and RPA, single-strand binding protein Msh4, the BLM helicase, and Mlh1/Mlh3. Rad51 and RPA are known to interact with single-stranded DNA regions poised for strand invasion. During wild-type meiosis in mouse, Rad51 and RPA are observed at $>300$ foci, apparently marking most or all positions of recombination initiation (Moens et al. 2002). Specificities of BLM and Msh4 foci are less understood; they are observed at later stages and somewhat lower numbers than Rad51 ( 200). While not specific for future crossovers in mouse, Msh4 co-localizes with the crossover-specific marker Mlh1, and is thus present at crossover sites (Moens et al. 2002).

Data from several organisms support the idea that ZMM proteins are required relatively early during meiotic recombination, possibly prior to completion of stable strand exchange. In the absence of Sycp1, early recombination markers, including Rad51, Msh4, and RPA foci, aberrantly persist into mouse pachytene, persist at around 100 chromosomal positions consistent with an early recombination defect (de Vries et al. 2005). Blocked sites likely include both designated COs and other sites, indicating that progression of both types of interaction depends on Sycp1 (de Vries et al. 2005).

In $C$. elegans, a single $\mathrm{CO}$ is formed on average along each of the six homolog pairs. In the absence of the C. elegans Zip3 homolog, Rad51 foci also accumulate transiently to twice the wild-type levels (Jantsch et al. 2004). In the absence of Msh4, Msh5, or Syp-2, >12 Rad51 foci persist beyond pachytene, suggesting a role of these proteins in progression of both designated COs and other recombination interactions at an early stage (Alpi et al. 2003, Colaiacovo et al. 2003).

Biochemical studies support the idea that the Mer3 helicase and the Msh4/Msh5 heterodimer promote or stabilize stable strand invasion (Figure 4). Purified Mer3 protein preferentially translocates along singlestranded DNA in the $3^{\prime}$ to $5^{\prime}$ direction to displace annealed DNA fragments up to 600 nucleotides in length in an ATP-dependent manner (Nakagawa et al. 2001). mer3 alleles carrying mutations in motifs important for helicase activity exhibit meiotic defects in DSB processing, CO formation, and homolog segregation similarly to the mer3 $\Delta$ deletion mutant, consistent with a role of DNA helicase activity in vivo (Nakagawa \& Kolodner 2002). Intriguingly, Mer3 does not function as an initiator of DNA strand exchange; rather, it stimulates Rad51-mediated DNA heteroduplex extension in the $3^{\prime} \rightarrow 5^{\prime}$ direction (Mazina et al. 2004). When a Rad51-coated, single-stranded DNA molecule is incubated with $5^{\prime}$ overlapping dsDNA, melting of dsDNA and heteroduplex extension is stimulated in the presence of Mer3 (Figure 4A). Heteroduplex extension in the opposite direction, by contrast, is specifically blocked, indicating that Mer3 promotes heteroduplex formation between invading strand and intact donor DNA, yet prevents reassociation of the original strands within the DSB (Mazina et al. 2004). This is precisely the activity required for stabilization of the first-strand invasion intermediate. Stable single-end invasion intermediates would thus not form in the absence of Mer3 due to its failure to stabilize these nascent interactions.

The Msh4/Msh5 heterodimer also exhibits activities that potentially stabilize strand invasion, consistent with the defect observed in the yeast msh5 $\Delta$ mutant (above). Purified human Msh4/Msh5 heterodimer binds to (single) Holliday junctions, but 
A

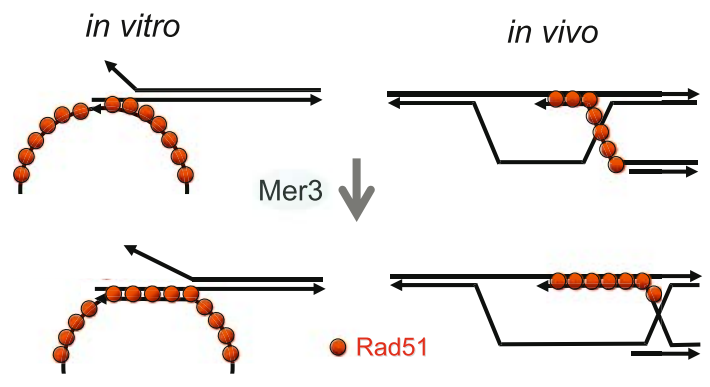

B

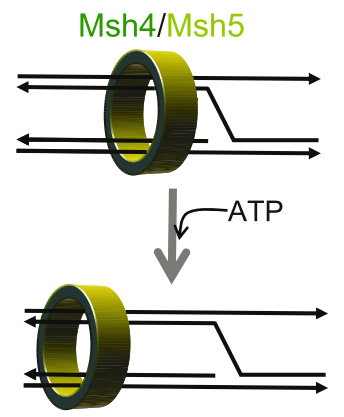

Figure 4. Biochemical functions of Mer3 and Msh4/Msh5. (A) Left panel: In vitro activity demonstrated for Mer3. Mer3 promotes strand exchange between a single-stranded DNA circle coated with Rad51 (orange circles) and a double-stranded linear DNA fragment carrying a $5^{\prime}$ overhang. When the double-stranded DNA fragment carries a 3' overhang, Rad51-promoted strand exchange is prevented (not shown). Right panel: In vivo function of Mer3 during stabilization of stable strand exchange analogous to its observed in vitro activities (for details see Mazina et al. 2004). (B) In vitro interaction of a human Msh4/Msh5 heterodimer with a progenitor Holliday junction. Msh4/Msh5 concurrently binds to two arms of Holliday junctions. ATP binding, but not hydrolysis result in sliding clamp activity of Msh4/Msh5 away from the point of strand exchange (Snowden et al. 2004).

not to mismatches or three-armed $\mathrm{Y}$ junctions (Snowden et al. 2004). Intriguingly, Msh4/Msh5 also binds to a three-armed progenitor Holliday junction, a substrate similar to the SEI strand invasion intermediate that is reduced in the $m s h 5 \Delta$ mutant (Figure 4B; Snowden et al. 2004, Börner et al. 2004). Following binding near the junction, Msh4/Msh5 is transformed into a sliding clamp, concurrently interacting with two DNA strands (Snowden et al. 2004). Loading of Msh4/Msh5 may thus stabilize the DNA structure associated with strand invasion. Given the proposed role of strand invasion in double Holliday junction geometry, the original invasion geometry may affect crossover-specific dHJ resolution (Storlazzi et al. 1996). Also, asynchronous resolution of tandem Holliday junctions may gener- ate single Holliday junction intermediates that can isomerize, isomerization could be prevented by binding of Msh4/Msh5. Detection of Msh4/Msh5 in yeast and mouse along pachytene chromosomes i.e. at a time when strand invasion has been completed raises the possibility of a later function of these proteins, e.g., during Holliday junction processing. Such functions would have eluded genetic analysis because in $m s h 5 \Delta$ deletion mutants recombination fails to progress to this point.

\section{ZMM proteins are dispensable for the interference decision and for formation of class II crossovers}

In yeast and Arabidopsis, substantial levels of COs are formed in the absence of ZMM proteins, revealing a class of ZMM-independent (class II) COs (see above). In yeast, levels of residual COs are affected by strain background and even incubation conditions. Crossovers form at $15 \%$ of wild-type levels in $z m m$ mutants at $33^{\circ} \mathrm{C}$ in the SK1 strain and at $30^{\circ} \mathrm{C}$ in the $\mathrm{BR}$ strain (see above). In the SK1 strain at low temperatures $\left(23^{\circ} \mathrm{C}\right)$, residual $\mathrm{CO}$ levels increase to $40-50 \%$ of wild-type in all $\mathrm{zmm}$ mutants, as suggested by physical analysis and genetic analysis of functional spores/gametes (e.g., Sym \& Roeder 1994, Nakagawa \& Ogawa 1999, Börner et al. 2004). NCOs also are aberrantly increased under these conditions (Börner et al. 2004).

Residual COs formed in $\mathrm{zmm}$ mutants at lower temperatures are defective in two respects: First, obligatory COs frequently fail to form, resulting in high levels of homolog nondisjunction (Sym \& Roeder 1994, Nakagawa \& Ogawa 1999, Novak et al. 2001). Second, residual COs do not exhibit crossover interference, as demonstrated by genetic analysis for zip1 $\Delta$ and mer3 4 (see below; Sym \& Roeder 1994, Nakagawa \& Ogawa 1999). In zip4 4 , residual COs formed under low-temperature conditions exhibit negative crossover interference, with COs in adjacent intervals forming at higher than expected frequencies (Tsubouchi et al. 2006). (Genetic analysis of residual COs is possible only under low-temperature conditions, since at high temperature $\left(33^{\circ} \mathrm{C}\right)$ and in the BR strain, zmm mutants undergo meiotic arrest.)

Residual COs in zmm mutants $m s h 4 \Delta$ and zipl $\Delta$ formed under low temperature conditions form without the appropriate controls, as indicated by 


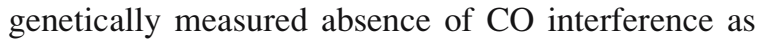

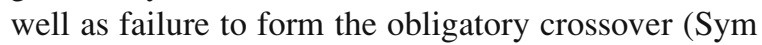

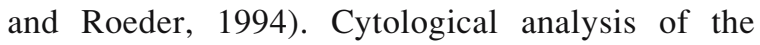

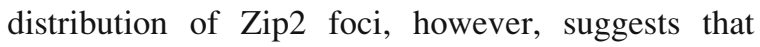

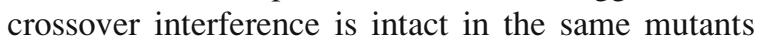

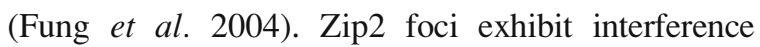

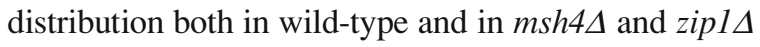

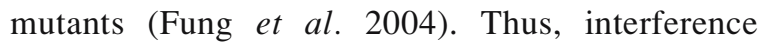

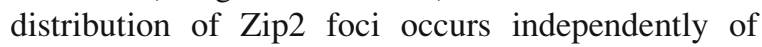

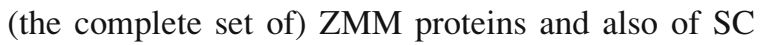

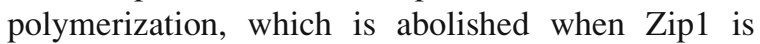

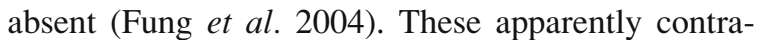

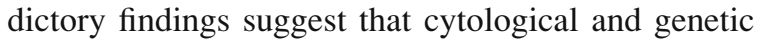

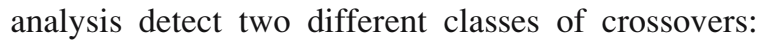

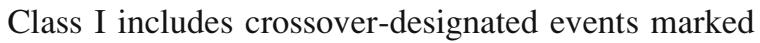

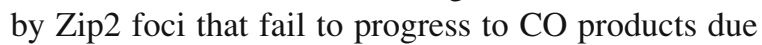

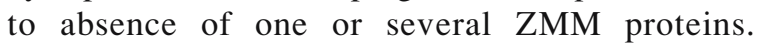

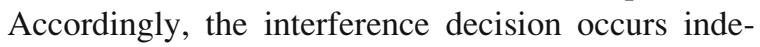

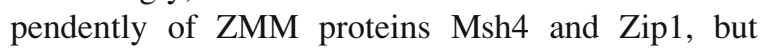

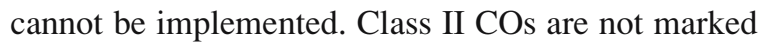

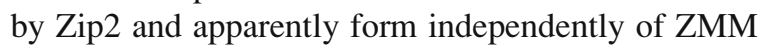

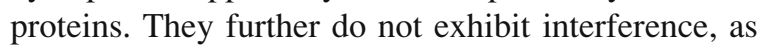

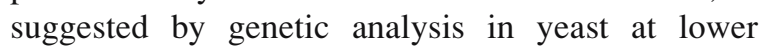

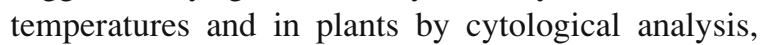

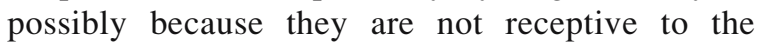

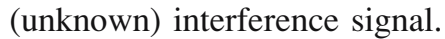

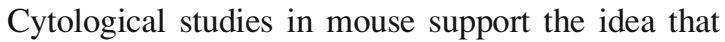

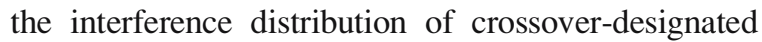

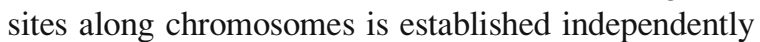

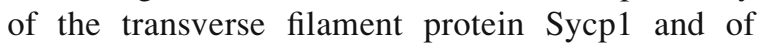

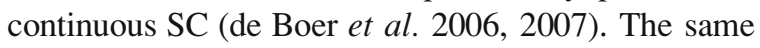

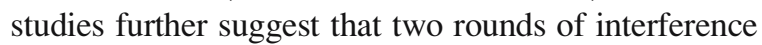

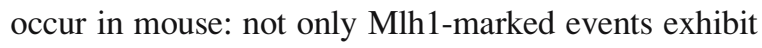

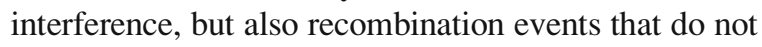

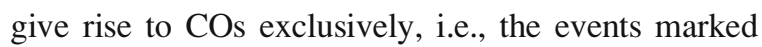

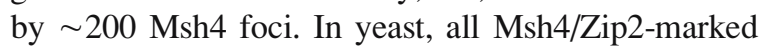

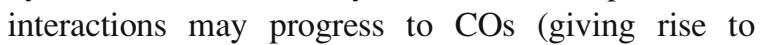

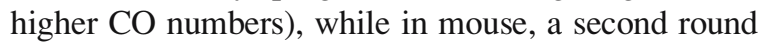

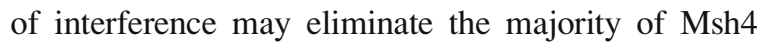

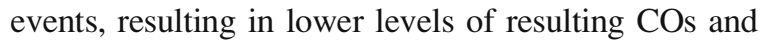

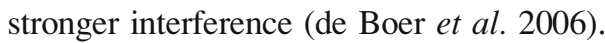

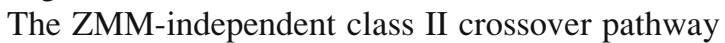

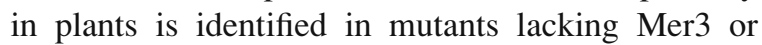

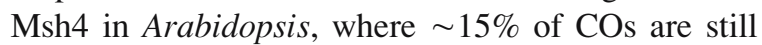

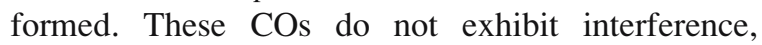

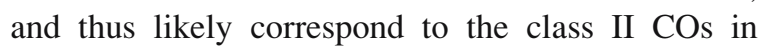

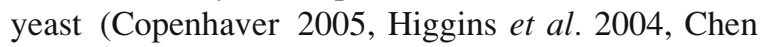

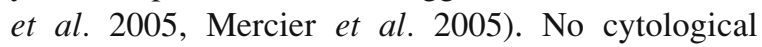

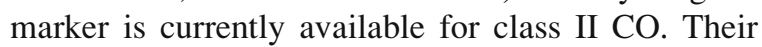

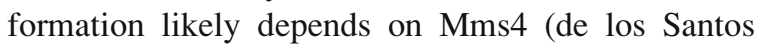

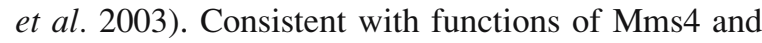

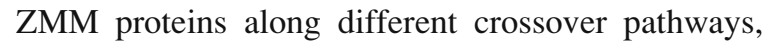

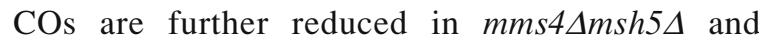

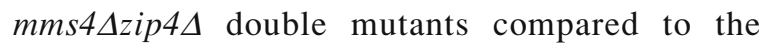

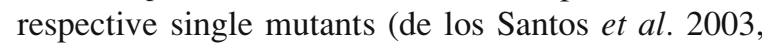

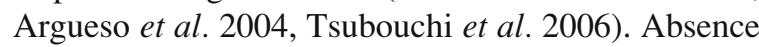

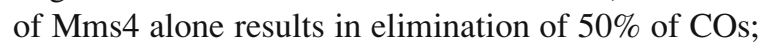

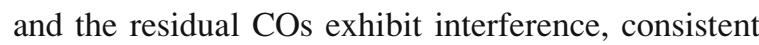

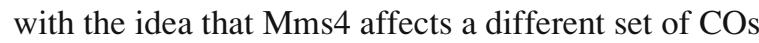

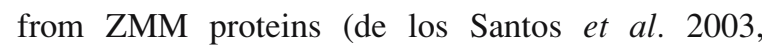

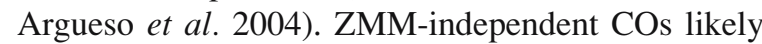

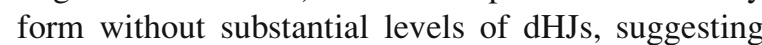

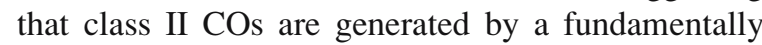

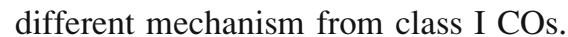

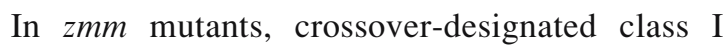

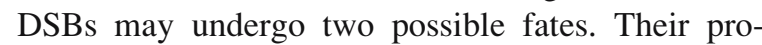

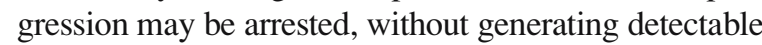

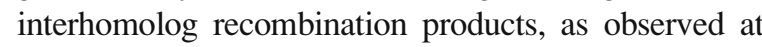

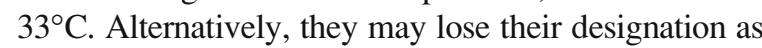

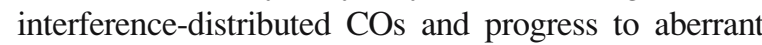

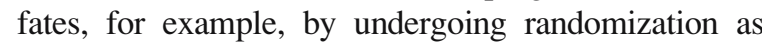

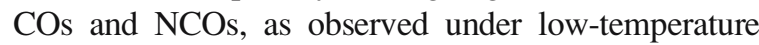

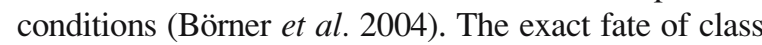

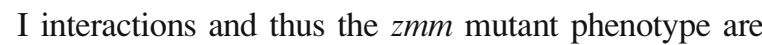

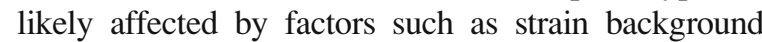

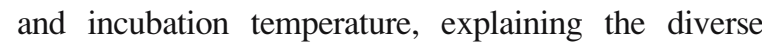

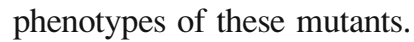

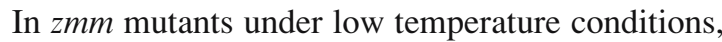

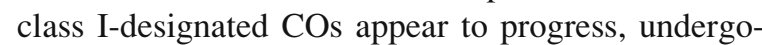

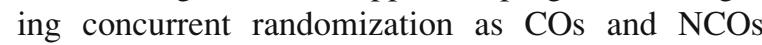

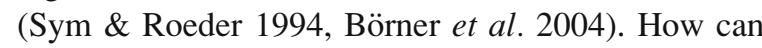

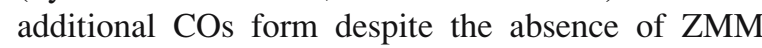

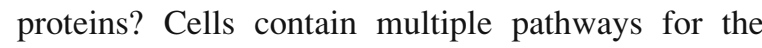

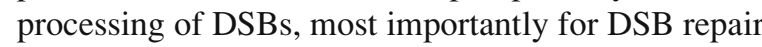

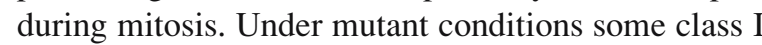

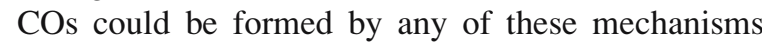

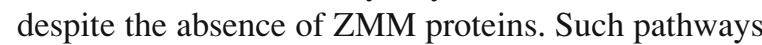

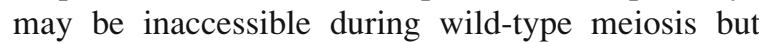

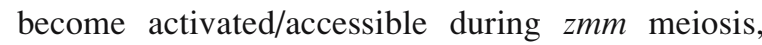

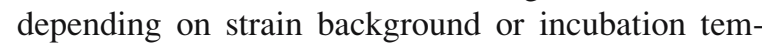

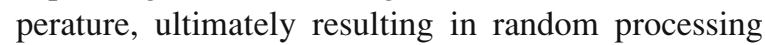

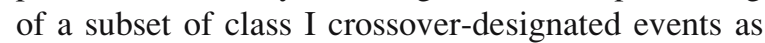

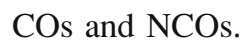

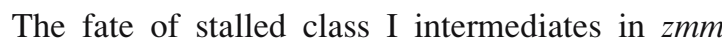

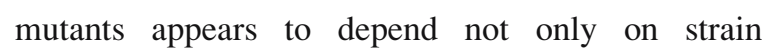

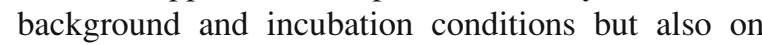

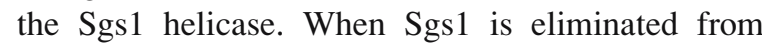

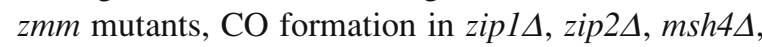

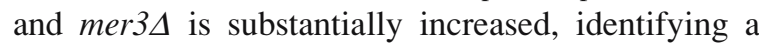

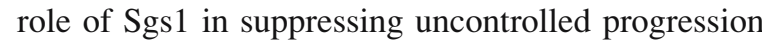


of recombination intermediates in the absence of ZMM proteins (Jessop et al. 2006).

\section{ZMM proteins collaborate in homolog synapsis}

During yeast meiosis, SC assembly and recombination are temporally and spatially closely associated: Assembly of the SC central element during zygotene occurs at the time when DSBs progress to SEIs (Padmore et al. 1991, Neil Hunter and G.V.B., unpublished data), and a similar temporal association may also exist in mouse (Mahadevaiah et al. 2001). Normal SC assembly critically depends on the formation of DSBs in a number of organisms, including yeast, mouse, and Arabidopsis (reviewed in de Boer \& Heyting 2006). In other organisms, such as C.elegans, SC can assemble even in mutants defective for DSB formation. Effects of $\mathrm{zmm}$ mutations on SC assembly have been investigated in both systems, providing further insights into likely ZMM protein functions.

Different ZMM proteins play fundamentally different roles in SC morphogenesis, based on their molecular function. The transverse filament protein Zip1 is an integral component of the SC (Sym et al. 1993). In the absence of Zip1, homologs fail to undergo close synapsis at $100 \mathrm{~nm}$, and instead remain co-aligned loosely at $400 \mathrm{~nm}$, with only a few close connections between axes called axial associations (Sym \& Roeder 1994). Zip1 likely plays an additional role of recombination early during synapsis, when homolog axes are first closely juxtaposed at the sites of recombination. Mer3 or Msh4/Msh5 likely mediate local recombination interactions directly, without participating in synapsis per se. Similarly, Zip2, Zip3, and Zip4, which are proposed to play a role in ubiquitin and/or SUMO protein modification (Cheng et al. 2005, Perry et al. 2005), likely perform local functions in protein modification at sites where they co-localize with Msh4/Msh5 (see below).

Despite apparently divergent molecular functions, mutant defects for SC assembly are remarkably similar between $\mathrm{zmm}$ mutants in yeast. Axial elements form normally, yet assembly of the SC central element is deficient at the leptotene-zygotene transition (Chua \& Roeder 1998, Agarwal and Roeder 2000, Börner et al. 2004). At the time of normal zygotene, partially synapsed nuclei are rare or absent in $\mathrm{zmm}$ mutants. Instead, Zip1 is present in irregular struc- tures corresponding to aberrant SC or early stages of polycomplex formation (Chua \& Roeder 1998, Börner et al. 2004). Polycomplexes are accumulations of SC components not associated with chromatin (Zickler \& Kleckner 1999). The synapsis defect in the zip $2 \Delta$ mutant is similar to that of zip1 $\Delta$, with a complete lack of EM detectable SC and axial association sites located between parallel, but unsynapsed, chromosome axes (Chua \& Roeder 1998). Zip1 polymerization occurs in this mutant, however, as detected by immunofluorescence (Chua \& Roeder 1998, Agarwal and Roeder 2000; Börner et al. 2004).

Functions of ZMM proteins for normal SC assembly have also been obseved in other organisms. In mouse, chromosome axes form normally without Msh4 or Msh5, yet the SC central element fails to assemble normally (de Vries et al. 1999, Edelmann et al. 1999, Kneitz et al. 2000). In Arabidopsis, again unsynapsed axes are detected in the absence of Msh4, although the synapsis defect is less severe than in mouse (Higgins et al. 2004). Some Mer3 mutations also results in defects in synapsis in Arabidopsis, but other alleles do not exhibit apparent synapsis defects despite defects in $\mathrm{CO}$ formation (Chen et al. 2005). No apparent defects in SC assembly have been observed in zip4 mutants in Arabidopsis, although such mutants exhibit severe recombination defects (Chelysheva et al. 2007).

In C. elegans, where SC forms independent of DSBs, ZMM proteins Msh4/Msh5 and Zhp3 are also dispensable for SC formation, although they do play roles in recombination (see above). Thus, at least in C. elegans, ZMM proteins apparently play a primary role in recombination and $\mathrm{zmm}$ mutant recombination defects are not the indirect effect of defective synapsis.

Some yeast ZMM proteins, including Zip3 and Zip4, also co-localize with Zip1 during early zygotene (Agarwal \& Roeder 2000, Henderson \& Keeney 2004, Tsubouchi et al. 2006). Some or all ZMM foci may thus represent sites from which SC polymerization initiates (Agarwal \& Roeder 2000). Based on this co-localization, (some) ZMM proteins have also been referred to as the synapsis initiation complex (SIC; Fung et al. 2004).

ZMM focus assembly has been studied in detail in yeast: During early zygotene, Zip3 and Zip4 localize to a few sites only, reaching their maximum numbers only during pachytene (Agarwal \& Roeder 2000, Tsubouchi et al. 2006). ZMM loading may thus 
occur with some asynchrony within a given nucleus. During pachytene, yeast ZMM proteins co-localize extensively, as suggested by co-localization between Zip2-Msh4, Zip2-Zip3, as well as Zip4-Zip2 (Agarwal \& Roeder 2000, Novak et al. 2001, Tsubouchi et al. 2006). Thus, most or all ZMM proteins load to the same sites along meiotic chromosomes.

Loading of yeast ZMM proteins into $60-70$ foci at pachytene depends on recombination initiation but also on the presence of other ZMM proteins. DSB formation is necessary for loading of all ZMM proteins to chromosomes in yeast (e.g., Chua \& Roeder 1998, Agarwal and Roeder 2000). Normal strand invasion, by contrast, appears dispensable for loading of at least some ZMM protein: In rad50S, which prevents resection and progression of DSBs, Zip2 loads apparently normally to chromosomes (Chua \& Roeder 1998). In the absence of the meiotic RecA homolog Dmc1, Zip2 also appears to load normally (Chua \& Roeder 1998). Resected DSBs accumulate in the respective strain background (Börner, unpublished), indicating that stable strand invasion is not required for Zip2 loading.

Mutant studies suggest complex mutual dependences in the loading of different ZMM proteins. Zip3 is required for loading of both Zip2 and Zip4/Spo22, but the latter proteins are not required for Zip3 loading (Tsubouchi et al. 2006). Zip2 and Zip4 loading are interdependent (Tsubouchi et al. 2006). Zip1 and Zip2 are required for loading at normal levels of Msh4 to chromosomes, while Zip2 loading occurs independently of Msh4 or Zip1 (Novak et al. 2001, Fung et al. 2004). Loading interdependences between Zip1 and Msh4 as well as Zip2 and Zip4 suggest that ZMM proteins may load not successively but collaboratively. While non-Zip1 ZMM proteins in yeast are required for the timely assembly of the synaptonemal complex, they are apparently not needed for the mechanics of Zip1 localization to chromosome axes. In yeast, but also in mouse and plants, the respective transverse filament proteins load onto chromosome axes abundantly, even in the absence of certain ZMM proteins. ZMM proteins may thus play a regulatory role in synapsis.

Mechanisms by which ZMM proteins affect each other's loading remain to be determined. It is also not known whether direct physical interactions exist between different ZMM proteins. Early loading ZMM proteins such as Zip3 may provide a loading platform for other proteins, as suggested by apparent interactions between Zip3 and Zip1 (Agarwal \& Roeder 2000). Alternatively, upstream proteins may mediate local transitions at recombination sites required for loading of additional ZMM proteins. Defective Zip2 loading in zip3A can be overcome by Zip2 overexpression (Agarwal \& Roeder 2000), which also improves Zip1 assembly, supporting the idea of a complex loading pattern rather than a simple linear pathway.

Molecular functions of ZMM proteins in synapsis are largely unknown. Such functions are particularly difficult to elucidate due to the close linkage between mutant defects in recombination and SC formation in both yeast and mouse. In yeast, numerous proteins with functions in meiotic DSB processing are required for axial element and/or SC central element assembly. In mutants that form DSBs efficiently but fail to resect them $(\mathrm{rad} 50 \mathrm{~S})$, formation of SC central element is severely defective despite apparently normal assembly of chromosome axes (see Zickler \& Kleckner 1999). Absence of the meiotic strand-exchange protein Dmc1 also delays SC assembly under certain conditions (Bishop et al. 1992). These observations suggest that recombination has to progress to a certain stage to license SC assembly. ZMM proteins may be required for SC formation in a similar way: ZMM proteins could promote recombination reactions at the stage of strand invasion, and SC assembly becomes possible only upon completion of such interactions. Recombination may bring homolog axes locally into sufficiently close proximity for transverse filament proteins to extend local areas of close juxtaposition into more global alignment (Börner et al. 2004). In this scenario, ZMM proteins (excluding transverse filament proteins) would affect synapsis via their role in recombination. Alternatively, ZMM proteins may play parallel roles in recombination and SC assembly. For example, ZMM proteins may primarily promote close juxtaposition of homolog axes around nascent recombination sites, thereby removing steric constraints that prevent recombination progression.

A mechanism by which Zip3 may effect SC polymerization has recently been proposed: Zip3 contains a RING finger which is present in known E3 components of ubiquitin- and/or SUMO-ligase enzyme systems (Perry et al. 2005, Cheng et al. 2006). Other components of the ubiquitin/SUMO transfer system include the E1 activation component and the E2 conjugation component. The E3 ligase 
transfers the small protein moiety to its ultimate target and thus provides substrate specificity (Kerscher et al. 2006). SUMOylated proteins are present in a largely continuous pattern along the synaptonemal complex, and Zip3 exhibits SUMOligase activity in vitro (Cheng et al. 2006, Hooker \& Roeder 2006). SC assembly is defective in the absence of the only yeast Ubc9 E2 SUMO conjugating component, consistent with a role of this protein modification in SC formation. Zip1 interacts with the yeast SUMO component Smt3, and SUMOylation was identified as a potential mechanism for targeting Zip1 to the homolog axes (Cheng et al. 2006). At the same time, it is noteworthy that a mutation in Ubc9, the only bona fide SUMO E2 in yeast, is defective for SC assembly but, unexpectedly, does not affect recombination in the same way as zip3 4 (Hooker \& Roeder 2006). If Zip3 and Ubc9 function along the same pathway, they should exhibit similar defects in recombination and SC formation. Zip3 may thus also exert other functions in addition to SUMO transfer (see Perry et al. 2005).

\section{Scenarios}

One of the intriguing questions about ZMM protein functions during meiosis is that of the relationship between SC assembly and recombination. Four scenarios appear feasible: (i) ZMM proteins play a primary role in recombination and SC assembly defects in $z m m$ mutants are a secondary consequence of the recombination defect; (ii) ZMM proteins primarily control SC assembly, and processing of recombination events past the DSB stage depends on the presence of local SC assembly; (iii) ZMM proteins affect recombination and SC assembly independently; and (iv) ZMM proteins mediate a coordinate transition in recombination and SC formation. While $\mathrm{zmm}$ mutants exhibit coordinate defects in both recombination and SC formation in mouse and $A$. thaliana, the situation is somewhat different in C. elegans, where SC forms independent of recombination. The situation in C. elegans, if transferable to other organisms, thus appears to exclude scenario (ii).

Work over recent years has identified roles of ZMM proteins in recombination and SC assembly, which are temporally and spatially closely associated. How could this close association be achieved?
Previously, it was proposed that recombination interactions are mounted on protein bridges between homologous chromosomes (Börner et al. 2004). Progression of such interactions may be controlled by steric constraints that may, for example, prevent stable strand invasion by keeping homologs at a distance, thus allowing unstable strand invasion, but no stable strand exchange. ZMM proteins may include DNA processing functions and mediators of protein interactions to concurrently effect strand invasion and modifications of the underlying interhomolog protein bridge. The Zip1 central element component may have become integrated with this complex to prevent stable strand invasion without prior availability of the main SC structural component, thereby ensuring that homolog synapsis can ensue as soon as the first crossover-specific recombination intermediate has been formed. Further work is required to understand the role of assembled $\mathrm{SC}$ in subsequent steps in recombination.

\section{Acknowledgements}

This work was supported by a Cleveland State University Startup grant and a Basil O'Connor Starter Scholar Research Award from the March of Dimes Foundation (\#5-FY06-581) to G.V.B.. Helpful suggestions from an anonymous reviewer are acknowledged.

\section{References}

Agarwal S, Roeder GS (2000) Zip3 provides a link between recombination enzymes and synaptonemal complex proteins. Cell 102: 245-255.

Allers T, Lichten M (2001) Differential timing and control of noncrossover and crossover recombination during meiosis. Cell 106: 47-57.

Alpi A, Pasierbek P, Gartner A, Loidl J (2003) Genetic and cytological characterization of the recombination protein RAD-51 in Caenorhabditis elegans. Chromosoma 112: 6-16.

Argueso JL, Wanat J, Gemici Z, Alani E (2004) Competing crossover pathways act during meiosis in Saccharomyces cerevisiae. Genetics 168: 1805-1816.

Bishop DK, Park D, Xu L, Kleckner N (1992) DMC1: a meiosisspecific yeast homolog of E. coli recA required for recombination, synaptonemal complex formation, and cell cycle progression. Cell 69: 439-456.

Bishop DK (1994) RecA homologs Dmc1 and Rad51 interact to form multiple nuclear complexes prior to meiotic chromosome synapsis. Cell 79: 1081-1092. 
Bishop DK, Zickler D (2004) Early decision; meiotic crossover interference prior to stable strand exchange and synapsis. Cell 117: 9-15.

Börner GV, Kleckner N, Hunter N (2004) Crossover/noncrossover differentiation, synaptonemal complex formation, and regulatory surveillance at the leptotene/zygotene transition of meiosis. Cell 117: $29-45$.

Carpenter AT (1987) Gene conversion, recombination nodules, and the initiation of meiotic synapsis. Bioessays 6: 232-236.

Chelysheva L, Gendrot G, Vezon D, et al. (2007) Zip4/Spo22 is required for class I CO formation but not for synapsis completion in Arabidopsis thaliana. PLoS Genet. 3:e83.

Chen C, Zhang W, Timofejeva L, Gerardin Y, Ma H (2005) The Arabidopsis ROCK-N-ROLLERS gene encodes a homolog of the yeast ATP-dependent DNA helicase MER3 and is required for normal meiotic crossover formation. Plant J 43: 321-334.

Cheng CH, Lo YH, Liang SS, et al. (2006) SUMO modifications control assembly of synaptonemal complex and polycomplex in meiosis of Saccharomyces cerevisiae. Genes Dev 20: 2067-2081.

Chua PR, Roeder GS (1998) Zip2, a meiosis-specific protein required for the initiation of chromosome synapsis. Cell 93: 349-359.

Colaiacovo MP, MacQueen AJ, Martinez-Perez E, et al. (2003) Synaptonemal complex assembly in $C$. elegans is dispensable for loading strand-exchange proteins but critical for proper completion of recombination. Dev Cell 5: 463-474.

Copenhaver GP (2005) Plant genetics: when not to interfere. Curr Biol. 15: R290-291.

de Boer E, Heyting C (2006) The diverse roles of transverse filaments of synaptonemal complexes in meiosis. Chromosoma 115: 220-234.

de Boer E, Dietrich AJ, Hoog C, Stam P, Heyting C (2007) Meiotic interference among MLH1 foci requires neither an intact axial element structure nor full synapsis. J Cell Sci 120: 731-736.

de Boer E, Stam P, Dietrich AJ, Pastink A, Heyting C (2006) Two levels of interference in mouse meiotic recombination. Proc Natl Acad Sci USA 103: 9607-9612.

de los Santos T, Hunter N, Lee C, Larkin B, Loidl J, Hollingsworth NM (2003) The Mus81/Mms4 endonuclease acts independently of double-Holliday junction resolution to promote a distinct subset of crossovers during meiosis in budding yeast. Genetics 164: 81-94.

de Vries SS, Baart EB, Dekker M, Siezen A, de Rooij DG, de Boer P, te Riele H (1999) Mouse MutS-like protein Msh5 is required for proper chromosome synapsis in male and female meiosis. Genes Dev 13: 523-531.

de Vries FA, de Boer E, van den Bosch M, et al. (2005) Mouse Sycp1 functions in synaptonemal complex assembly, meiotic recombination, and XY body formation. Genes Dev 19: 1376-1389.

Edelmann W, Cohen PE, Kneitz B, et al. (1999) Mammalian MutS homologue 5 is required for chromosome pairing in meiosis. Nat Genet 21: 123-127.

Fung JC, Rockmill B, Odell M, Roeder GS (2004) Imposition of crossover interference through the nonrandom distribution of synapsis initiation complexes. Cell 116: 795-802.

Guillon H, Baudat F, Grey C, Liskay RM, de Massy B (2005) Crossover and noncrossover pathways in mouse meiosis. Mol Cell 20: 563-573.

Hassold T, Hunt P (2001) To err (meiotically) is human: the genesis of human aneuploidy. Nat Rev Genet 2: 280-291.
Henderson KA, Keeney S (2004) Tying synaptonemal complex initiation to the formation and programmed repair of DNA double-strand breaks. Proc Natl Acad Sci USA 101: 4519-4524.

Higgins JD, Armstrong SJ, Franklin FC, Jones GH (2004) The Arabidopsis MutS homolog AtMSH4 functions at an early step in recombination: evidence for two classes of recombination in Arabidopsis. Genes Dev 18: 2557-2570.

Higgins JD, Sanchez-Moran E, Armstrong SJ, Jones GH, Franklin FC (2005) The Arabidopsis synaptonemal complex protein ZYP1 is required for chromosome synapsis and normal fidelity of crossing over. Genes Dev 19: 2488-2500.

Hollingsworth NM, Ponte L, Halsey C (1995) MSH5, a novel MutS homolog, facilitates meiotic reciprocal recombination between homologs in Saccharomyces cerevisiae but not mismatch repair. Genes Dev 9: 1728-1739.

Hooker GW, Roeder GS (2006) A role for SUMO in meiotic chromosome synapsis. Curr Biol 16: 1238-1243.

Hunter N, Kleckner N (2001) The single-end invasion: an asymmetric intermediate at the double-strand break to doubleHolliday junction transition of meiotic recombination. Cell 106: 59-70.

Jantsch V, Pasierbek P, Mueller MM, Schweizer D, Jantsch M, Loidl J (2004) Targeted gene knockout reveals a role in meiotic recombination for ZHP-3, a Zip3-related protein in Caenorhabditis elegans. Mol Cell Biol 24: 7998-8006.

Jessop L, Rockmill B, Roeder GS, Lichten M (2006) Meiotic chromosome synapsis-promoting proteins antagonize the anticrossover activity of sgs1. PLoS Genet. 2: e155.

Kelly KO, Dernburg AF, Stanfield GM, Villeneuve AM (2000) Caenorhabditis elegans msh-5 is required for both normal and radiation-induced meiotic crossing over but not for completion of meiosis. Genetics 156: 617-630.

Kerscher O, Felberbaum R, Hochstrasser M (2006) Modification of proteins by ubiquitin and ubiquitin-like proteins. Annu Rev Cell Dev Biol 22: 159-180.

Kneitz B, Cohen PE, Avdievich E, et al. (2000) MutS homolog 4 localization to meiotic chromosomes is required for chromosome pairing during meiosis in male and female mice. Genes Dev 14: 1085-1097.

Lynn A, Ashley T, Hassold T (2004) Variation in human meiotic recombination. Annu Rev Genomics Hum Genet 5: 317-349.

MacQueen AJ, Colaiacovo MP, McDonald K, Villeneuve AM (2002) Synapsis-dependent and -independent mechanisms stabilize homolog pairing during meiotic prophase in C. elegans. Genes Dev 16: 2428-2442.

Mahadevaiah SK, Turner JM, Baudat F, et al. (2001) Recombinational DNA double-strand breaks in mice precede synapsis. Nat Genet 27: 271-276.

Marcon E, Moens P (2003) MLH1p and MLH3p localize to precociously induced chiasmata of okadaic-acid-treated mouse spermatocytes. Genetics 165: 2283-2287.

Martini E, Diaz RL, Hunter N, Keeney S (2006) Crossover homeostasis in yeast meiosis. Cell 126: 285-295.

Mazina OM, Mazin AV, Nakagawa T, Kolodner RD, Kowalczykowski SC (2004) Saccharomyces cerevisiae Mer3 helicase stimulates $3^{\prime}-5^{\prime}$ heteroduplex extension by Rad51; implications for crossover control in meiotic recombination. Cell 117: 47-56. 
Mercier R, Jolivet S, Vezon D, et al. (2005) Two meiotic crossover classes cohabit in Arabidopsis: one is dependent on MER3, whereas the other one is not. Curr Biol 15: 692-701.

Mitra N, Roeder GS (2007) A novel non-null ZIP1 allele triggers meiotic arrest with synapsed chromosomes in $S$. cerevisiae. Genetics 176: 773-787.

Moens PB, Kolas NK, Tarsounas M, Marcon E, Cohen PE, Spyropoulos B (2002) The time course and chromosomal localization of recombination-related proteins at meiosis in the mouse are compatible with models that can resolve the early DNA-DNA interactions without reciprocal recombination. $J$ Cell Sci 15: 1611-1622.

Nakagawa T, Kolodner RD (2002) Saccharomyces cerevisiae Mer3 is a DNA helicase involved in meiotic crossing over. Mol Cell Biol 22: 3281-3291.

Nakagawa T, Flores-Rozas H, Kolodner RD (2001) The MER3 helicase involved in meiotic crossing over is stimulated by single-stranded DNA-binding proteins and unwinds DNA in the $3^{\prime}$ to $5^{\prime}$ direction. $J$ Biol Chem 276: 31487-31493.

Nakagawa T, Ogawa H (1999) The Saccharomyces cerevisiae MER3 gene, encoding a novel helicase-like protein, is required for crossover control in meiosis. EMBO J 18: 5714-5723.

Neyton S, Lespinasse F, Moens PB, et al. (2004) Association between MSH4 (MutS homologue 4) and the DNA strandexchange RAD51 and DMC1 proteins during mammalian meiosis. Mol Hum Reprod 10: 917-924.

Novak JE, Ross-Macdonald PB, Roeder GS (2001) The budding yeast Msh4 protein functions in chromosome synapsis and the regulation of crossover distribution. Genetics 158: 1013-1025.

Padmore R, Cao L, Kleckner N (1991) Temporal comparison of recombination and synaptonemal complex formation during meiosis in S. cerevisiae. Cell 66: 1239-1256.

Page SL, Hawley RS (2004) The genetics and molecular biology of the synaptonemal complex. Annu Rev Cell Dev Biol 20: 525-558.

Perry J, Kleckner N, Börner GV (2005) Bioinformatic analyses implicate the collaborating meiotic crossover/chiasma proteins Zip2, Zip3, and Spo22/Zip4 in ubiquitin labeling. Proc Natl Acad Sci USA 102: 17594-17599.

Rockmill B, Fung JC, Branda SS, Roeder GS (2003) The Sgs1 helicase regulates chromosome synapsis and meiotic crossing over. Curr Biol 13: 1954-1962.
Schwacha A, Kleckner N (1995) Identification of double Holliday junctions as intermediates in meiotic recombination. Cell $\mathbf{8 3}$ : 783-791.

Schwacha A, Kleckner N (1997) Interhomolog bias during meiotic recombination: meiotic functions promote a highly differentiated interhomolog-only pathway. Cell 90: 1123-1135.

Snowden T, Acharya S, Butz C, Berardini M, Fishel R (2004) hMSH4-hMSH5 recognizes Holliday junctions and forms a meiosis-specific sliding clamp that embraces homologous chromosomes. Mol Cell 15: 437-451.

Storlazzi A, Xu L, Schwacha A, Kleckner N (1996) Synaptonemal complex (SC) component Zip1 plays a role in meiotic recombination independent of SC polymerization along the chromosomes. Proc Natl Acad Sci USA 93: 9043-9048.

Sun H, Treco D, Schultes NP, Szostak JW (1989) Double-strand breaks at an initiation site for meiotic gene conversion. Nature 338: $87-90$.

Sym M, Engebrecht JA, Roeder GS (1993) ZIP1 is a synaptonemal complex protein required for meiotic chromosome synapsis. Cell 72: $365-378$.

Sym M, Roeder GS (1994) Crossover interference is abolished in the absence of a synaptonemal complex protein. Cell 79: 283-292.

Tanaka K, Miyamoto N, Shouguchi-Miyata J, Ikeda JE (2006) HFM1, the human homologue of yeast Mer3, encodes a putative DNA helicase expressed specifically in germ-line cells. DNA Seq 17: 226-242.

Tease C, Hartshorne GM, Hulten MA (2002) Patterns of meiotic recombination in human fetal oocytes. Am J Hum Genet 70: 1469-1479.

Tsubouchi T, Zhao H, Roeder GS (2006) The meiosis-specific Zip4 protein regulates crossover distribution by promoting synaptonemal complex formation together with Zip2. Dev Cell 10: 809-819.

Zalevsky J, MacQueen AJ, Duffy JB, Kemphues KJ, Villeneuve AM (1999) Crossing over during Caenorhabditis elegans meiosis requires a conserved MutS-based pathway that is partially dispensable in budding yeast. Genetics 153: 1271-1283.

Zickler D, Kleckner N (1999) Meiotic chromosomes: integrating structure and function. Annu Rev Genet 33: 603-754. 